\title{
Time-frequency analysis of the sea state with the Andrea freak wave
}

\section{Z. Cherneva and C. Guedes Soares}

Centre for Marine Technology and Engineering (CENTEC), Instituto Superior Técnico, Universidade de Lisboa, Av. Rovisco Pais, 1049-001 Lisbon, Portugal

Correspondence to: C. Guedes Soares (c.guedes.soares@centec.tecnico.ulisboa.pt)

Received: 21 August 2013 - Published in Nat. Hazards Earth Syst. Sci. Discuss.: 14 February 2014

Revised: 17 September 2014 - Accepted: 10 October 2014 - Published: 1 December 2014

\begin{abstract}
The nonlinear and nonstationary properties of a special field wave record are analysed with the Wigner spectrum with the Choi-Williams kernel. The wave time series, which was recorded at the Ekofisk complex in the central North Sea at 00:40 UTC (universal time coordinated) on 9 November 2007, contains an abnormally high wave known as the "Andrea" wave. The ability of the Wigner spectrum to reveal the wave energy distribution in frequency and time is demonstrated. The results are compared with previous investigations for different sea states and also the state with Draupner's abnormal "New Year" wave.
\end{abstract}

\section{Introduction}

Several events in which ships have been damaged by giant waves have been reported in the literature during the last 20 years (e.g. Kjeldsen, 1997; Faulkner and Buckley, 1997). The interest in this type of waves have lead to the analysis of data from real sea states, i.e. looking for occurrences of abnormally high waves, which have been reported in an increasing number of works. Instrumental records have identified this type of wave in the North Sea (Skourup et al., 1996; Haver and Andersen, 2000; Wolfram et al., 2000; Guedes Soares et al., 2003; Magnusson and Donelan, 2013), the Sea of Japan (Yasuda and Mori, 1997; Mori et al., 2002), the Gulf of Mexico (Guedes Soares et al., 2004) and even in the Baltic Sea (Didenkulova, 2011). A more comprehensive review of registered huge waves is made in Kharif et al. (2009).

Waves of anomalously large size, called abnormal, freak, or rogue waves, are very steep in the last stage of their evolution and propagate as a wall of water. A typical abnormal wave is a single event with a characteristic lifetime of just a few seconds. Before breaking, it has a crest 3-4 times higher than the crests of neighbouring waves and appears almost instantly, as it has been identified in wave records. There is no doubt that such waves are essentially of nonlinear character and can be generated by different mechanisms (Kharif et al., 2009).

Understanding the nature of abnormal waves can be achieved by a detailed analysis of the time records of free surface elevation using different methods to determine their time-frequency energy distributions.

The standard Fourier analysis is a powerful tool for the investigation of waves because it provides the opportunity to decompose the series into individual frequency components and to specify their relative intensity. However, the usual frequency spectrum does not give any information when these frequencies bring a great amount of energy. The assumption is that it happens in the same manner during all periods of measurement.

Visual observation of the sea surface shows that the wind waves of various heights move in reiterated groups of different length and number of waves. This means that for short time intervals nearly equal to the group duration there exists significant change of the wave energy per unit square surface. Therefore, the wind wave for short intervals of time is not a stationary process as is usually supposed.

The development of methods for investigation of the spectrum changes in time begins with several classical works (Gabor, 1946; Ville, 1948; Page, 1952) that developed the idea of Wigner (Wigner, 1932) to take into account the quantum corrections for thermodynamic equilibrium. These studies mark the beginning of the fundamental analysis and 
explanation of the physical and mathematical ideas for understanding what is changeable in the time spectrum. The main idea is to find a joint function of frequency and time that describes the energy distribution of the process. In the ideal case such distribution will be used and transformed in a same manner as any joint distribution. Nevertheless, the joint functions in frequency and time of the wave energy are not distributions in a probabilistic sense because the time-frequency spectra provide a distribution of the energy while probabilistic joint distribution of two variables describes their joint probability. However, for historical reasons the time-frequency spectra of the signals are often also called "distributions".

The previously mentioned authors lead their works by a partial mathematical similarity with some problems in quantum mechanics and signal processing. It is necessary to highlight clearly that the analogy between quantum mechanics and the theory of signals is formal and that the physical interpretation in these two branches of knowledge is completely different (Cohen, 1995).

First, Gabor (1946) developed the mathematical basis of the time-frequency method and introduced a very important concept for analytical signal that is later applied in radiophysics and the theory of signals. Furthermore, (Turner, 1954; Levin, 1967) it is shown that using procedures similar to those in the work of Page (1952) it is possible to find many other time-frequency distributions. Rihaczek (1968) deduced a new distribution examining problems of physics connected with signals spreading. A lot of ideas about the convolution and filtering of the nonstationary stochastic processes are introduced in the detailed work of Mark (1970) and are now applied in the everyday practice of many scientific fields.

The last 2 decades of the 20th century mark a great progress of investigations dedicated to the time-frequency structure of the signals. This revival of the scientific interest (Claasen and Meklenbruker, 1980a, b, c; Janse and Kaizer, 1983 ) is accompanied with the development of unique ideas of the spectral distributions and demonstrates their practical application. Moreover, it begins to clarify the similarity and difference between the quantum mechanics and the theory of the real signals. In the works of Boashash and Whitehouse (1986) and Boles and Boashash (1988), the ideas developed by Claasen and Meeklenbräuker (1980a, b, c) are implemented, possibly for the first time, to deal with the real problems of geophysics.

In the course of time a new manner of thinking arose for the interpretation and use of the time-frequency energy distributions. Different points of view and different scientific interests extend the understanding of what the nonstationary processes are and diversify the techniques of their investigation. A good review of the applied and scientific problems connected with time-frequency analysis can be found in Cohen (1995), Hlawatsch and Boudreaux-Bartels (1992),
Boashash (1991), Meecklenbräuker (1985), Hlawatsch and Flandrin (1992) and Huang et al. (1998).

Some of time-frequency energy distributions are already used to study the nature of the ocean wind waves such as wavelet distribution (Liu, 1994, 2000 a, b; Massel, 2001), spectrogram distribution (Guedes Soares and Cherneva, 2005; Cherneva and Guedes Soares, 2005), the empirical mode decomposition (Huang et al., 1998; Schurlman, 2001; Veltcheva and Guedes Soares, 2007, 2011) and Wigner spectra (Cherneva and Guedes Soares, 2008, 2011, 2012).

The present study analyses sea state wave data from single point measurements, in order to characterize the nonstationary and nonlinear properties of the large waves registered in the North Sea at the Andrea platform. It is a followup to a series of studies performed (Guedes Soares et al., 2003, 2004; Guedes Soares and Cherneva, 2005; Cherneva and Guedes Soares, 2008, 2011, 2012; Veltcheva and Guedes Soares, 2007, 2011). In what follows, Sect. 2 provides a theoretical background to presenting wind waves as an analytical process, introduces a definition of a Wigner spectrum and briefly describes the Benjamin-Feir instability. Results and discussions are the subject of Sect. 3. Conclusions are made in Sect. 4.

\section{Theoretical background}

\subsection{Wind waves as an analytical process}

It was already mentioned that the concept for the analytical signal belongs to Gabor (1946). After the pioneering work of Longuet-Higgins (1952), mathematical and physical methods from radiophysics, theory of noise and signal processing are widely introduced in the investigation of wind waves (e.g. Bitner, 1980; Bitner-Gregersen and Gran, 1983; Tayfun and Lo, 1989).

If $\eta(t)$ is the surface elevation and $\dot{\eta}(t)$ is its Hilbert transform, the complex process $x(t)=\eta(t)+j \dot{\eta}(t)$ is an analytical process, corresponding to $\eta(t)$. The process $x(t)$ can be presented also as $x(t)=|A(t)| \exp [j \psi(t)]$, where the envelope $|A(t)|$ and the phase $\psi(t)$ are defined as

$$
\begin{aligned}
& |A(t)|=\left[\dot{\eta}^{2}(t)+^{2}(t)\right]^{1 / 2}, \\
& \psi(t)=\operatorname{arctg}[\dot{\eta}(t) / \eta(t)] .
\end{aligned}
$$

The phase $\psi(t)$ can also be written as

$\psi(t)=\omega_{0} t+\varphi(t)$,

where $\omega_{0}$ is co-called carrier frequency and $\varphi(t)$ is the local phase. The time derivative of the phase function $\psi(t)$ defines a local frequency of the time series:

$\omega(t)=\mathrm{d} \psi / \mathrm{d} t=\omega_{0}+\mathrm{d} \varphi(t) / \mathrm{d} t$. 
In this sense, the local frequency $\omega(t)$ is a rate of change of the phase (Van der Pol, 1946). If it is assumed that at each time instant $t$ there exists only one single frequency component the local frequency is defined also as an average frequency at a particular time. Then the sea state is restricted to a single mean wave characteristic, such as the local frequency, which changes in time.

In Eq. (3) the unwrapped phase function $\psi(t)$ consists of a linear part $\omega_{0} t$ increasing with time and a deviation part $\varphi(t)$ superimposed on $\omega_{0} t$. Therefore, from the slope of the unwrapped phase function $\psi(t)$ it is possible to find the carrier frequency $\omega_{0}$. It is obvious that the carrier frequency $\omega_{0}$ does not coincide with the spectrum peak frequency $\omega_{\mathrm{p}}$. Usually $\omega_{\mathrm{p}}$ is less than $\omega_{0}$ for the real sea state spectra and the fact is not connected with downshifting of the spectrum peak frequency due to Benjamin-Feir instability. Only when waves have nearly equal phases during a given time interval and the change of the phases in time $\theta=\mathrm{d} \varphi(t) / \mathrm{d} t$ is small, the local frequency is equal to the carrier frequency $\omega_{0}$. If the phase change has negative slope $\theta=\mathrm{d} \varphi(t) / \mathrm{d} t<0$, then the local frequency is lower than the carrier one. A detailed discussion about the local frequency of the signal can be found in the classic work of Cohen (1989).

\subsection{Wigner spectrum}

For the analytical signal $x(t)$, Fonolosa and Nikias (1993, 1994) define the Wigner high-order time-frequency spectrum as

$$
\begin{aligned}
& W_{k x}\left(t, f_{1}, \ldots, f_{k}\right)=\iiint_{\Omega u \tau_{1}} \ldots \int_{\tau_{k}} \Phi\left(\Omega, \tau_{1}, \ldots, \tau_{k}\right) \\
& \quad \times R_{k u}\left(\tau_{1}, \ldots, \tau_{k}\right) \exp (2 \pi j u \Omega) \exp (-2 \pi j t \Omega) \\
& \quad \times \prod_{i=1}^{k} \exp \left(-j 2 \pi f_{i} \tau_{i}\right) \mathrm{d} \tau_{i} \mathrm{~d} u \mathrm{~d} \Omega .
\end{aligned}
$$

Here $W_{k x}\left(t, f_{1}, \ldots, f_{k}\right)$ is a $k$-dimensional Fourier transform of a $k$-dimensional local moment function $R_{k u}\left(\tau_{1}, \ldots, \tau_{k}\right)$, and $\Phi\left(\Omega, \tau_{1}, \ldots, \tau_{k}\right)$ is a kernel introduced to reduce the aliasing. The definition (Eq. 5) is preferred because it allows for an opportunity to estimate the higher-order time-frequency spectra of the waves as it was done before for the "New Year wave" (Cherneva and Guedes Soares, 2008).

For $k=1$, Eq. (5) leads to a definition of the Wigner spectrum. The most applied time-frequency Wigner spectra can be obtained using different kernels $\Phi(\Omega, \tau)$. In particular, the Wigner-Ville distribution corresponds to $\Phi(\Omega, \tau)=1$ and the Rihaczek distribution to $\Phi(\Omega, \tau)=\exp (j \pi \tau \Omega) \quad$ (Cohen, 1966). Here the Choi-Williams kernel $\Phi(\Omega, \tau)=\exp \left(-\Omega^{2} \tau^{2} / \sigma\right)$ is used, where $\sigma=0.05$ (Choi and Williams, 1989).

\subsection{Benjamin-Feir instability}

Ocean waves in their nature are nonlinear and dispersive waves. Experimental studies of Benjamin and Feir (1967) show that regular wave trains in deep water are responsible for a number of instabilities now known as Benjamin-Feir instability. The instability is a result of an interaction between three monochromatic wave trains: carrier $\omega_{\mathrm{c}}$, upper $\omega_{+}$and lower $\omega_{-}$sideband waves. According to a perturbation analysis accomplished by Benjamin and Feir (1967) based on the Euler equations, if $\delta \omega$ is a small frequency perturbation, then the carrier wave and the sideband waves have to meet the requirements to four wave-resonance conditions for infinitesimal waves where

$\omega_{ \pm}=\omega_{\mathrm{c}} \pm \delta \omega$,
$2 \omega_{\mathrm{c}}=\omega_{+}+\omega_{-}$,
$2 k_{\mathrm{c}}=k_{+}+k_{-}+\Delta k$.

In Eq. (6) the frequencies and the wave numbers are connected by the linear dispersion relationship in deep water and $\Delta k$ is a small mismatch. The group of three waves with initial carrier wave amplitude $a_{\mathrm{c}}$ is unstable if the inequality $0<\hat{\delta} \leq \sqrt{2}$ is satisfied, where $\hat{\delta}=\delta \omega / \varepsilon \omega_{\mathrm{c}}$ and $\varepsilon=a_{\mathrm{c}} k_{\mathrm{c}}$. Then the sideband waves begin to grow in amplitude at the expense of the main wave. The maximum $\beta_{x}=\mathrm{d}(\ln a) / \mathrm{d}(k x)$ appears when $\hat{\delta}=1.0$ and the initial phases of the sidebands are $\psi_{ \pm}=-\pi / 4$. This theory is in good agreement with observations for wave steepness $\varepsilon$ in the interval [0.07, 0.17] (Benjamin, 1967).

The evolution of a nonlinear wave train without dissipation manifests the so-called Fermi-Pasta-Ulam phenomenon: periodically increasing and decreasing the modulation causing the wave to return to its initial form (Lake et al., 1977). Furthermore, Longuet-Higgins (1978) found that subharmonic instabilities of the Benjamin-Feir type are restricted to waves whose steepness $\varepsilon=a k$ has an upper limit. As $\varepsilon$ increases beyond 0.346 the wave modes become stable again.

Tulin and Waseda (1999) compared their experimental data with the theoretical predictions of Benjamin and Feir (1967) and Krasitskii (1994). They found that Krasitskii's modification of the Zakharov evolution equation (Zakharov, 1968) correctly predicts the major features of the energy increase in the lower sideband relative to the upper sideband. Additionally, they argued that downshifting to a lower sideband of the spectral peak also appears in the absence of breaking, and showed the significant role of the balance between momentum losses and energy dissipation in the exchange of energy between the sidebands.

A detailed review on the subject can be found in the works of Yuen and Lake (1980), Hammack and Henderson (1993), Kharif et al. (2009), and Massel (2010). 
Table 1. Parameters characterizing the nonlinearity of the series and of the individual freak waves.

\begin{tabular}{lrcccccccc}
\hline Quantity & $H_{\mathrm{s}}, \mathrm{m}$ & $k_{\mathrm{p}} h$ & $\varepsilon=a_{\mathrm{s}} k_{\mathrm{p}}$ & $a_{\mathrm{s}} / h$ & $H_{\text {freak }}, \mathrm{m}$ & $T_{\text {freak }}, \mathrm{s}$ & $\varepsilon_{\text {freak }}$ & $k_{\text {freak }} h$ & $a_{\text {freak }} / h$ \\
\hline Andrea & 9.18 & 1.61 & 0.106 & 0.066 & 21.14 & 12.0 & 0.295 & 1.9543 & 0.151 \\
Draupner & 11.92 & 1.96 & 0.081 & 0.085 & 25.01 & 13.1 & 0.293 & 1.640 & 0.179 \\
\hline
\end{tabular}

\section{Results and discussion}

Here a wave time series measured at 00:40 UTC (universal standard time) on 9 November 2007 is analysed. The series containing a huge wave named the "Andrea" rogue wave was recorded at the Ekofisk complex operated by ConocoPhillips in the central North Sea $\left(56^{\circ} 30^{\prime} \mathrm{N}, 3^{\circ} 12^{\prime} \mathrm{E}\right)$, where the water depth is between 70 and $80 \mathrm{~m}$. A detailed description of the weather conditions, measuring installations and some comparisons between the Draupner's New Year wave and the Andrea wave can be found in Magnusson and Donelan (2013). Table 1 shows some of the data in that paper with some of the present results.

Table 1 presents the three parameters characterizing the nonlinearity of the series with freak waves measured at the Draupner and the Andrea platforms: $k_{\mathrm{p}} h, \varepsilon=a_{\mathrm{s}} k_{\mathrm{p}}$ and $a_{\mathrm{s}} / h$, where $a_{\mathrm{s}}=H_{\mathrm{s}} / 2, h$ is the depth, $H_{\mathrm{s}}$ is the significant wave height and $k_{\mathrm{p}}$ is the wave number of the spectrum peak frequency. Similar parameters but for the individual abnormal waves, noted with the subscript "freak", are also calculated, where $H_{\text {freak }}$ is the height of the freak wave and $a_{\text {freak }}=H_{\text {freak }} / 2 ; T_{\text {freak }}$ is its individual period and $k_{\text {freak }}$ is the wave number corresponding to that period. The steepness of the series is 0.081 for the New Year wave and 0.106 for the Andrea wave. The individual steepness of the rogue waves is $\varepsilon_{\text {freak }} \approx 0.3$, which is less than the upper limit of 0.346 (Longuet-Higgins, 1978). For freak waves the parameters $k_{\text {freak }} h \approx 2$, and $a_{\text {freak }} / h \approx 0.2$; from this it can be concluded that the registered two abnormal waves are strongly nonlinear (Kurkin and Pelinovsky, 2004; Cherneva and Guedes Soares, 2008).

The available Andrea time series has 4600 registered ordinates with a $5 \mathrm{~Hz}$ sampling frequency that gives $15 \mathrm{~min}$ durations of the registration presented in Fig. 1a. The existence of foam or possible breaking is taken into account as it is described in Magnusson and Donelan (2013). The series is too short to derive any conclusion about the distribution of the wave heights or crests as done in Cherneva et al. (2009, 2013). Because of that, in this work the study is limited to nonlinear and nonstationary properties of the waves.

There are three high-wave groups which are interesting and investigated here: the first one is a long group in the time interval of 560-720 s containing a huge wave in its beginning; the second group exists in the interval of $420-540 \mathrm{~s}$; waves in the third group of the interval of 130-200 s are high, as the waves in the second group, but do not have the typical triangle structure as in the first two groups. In Fig. 1a the

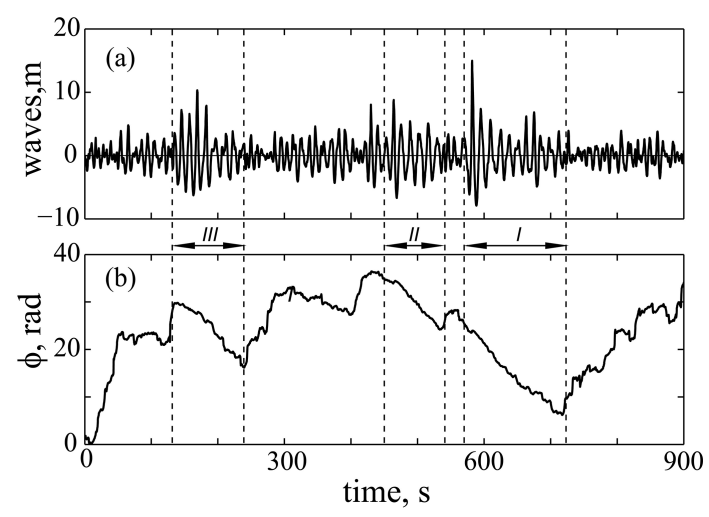

Figure 1. Andrea freak wave. (a) Series measured at 00:40 UTC on 9 November 2007; (b) phase $\varphi$ in time.

groups are separated from the rest of the series by vertical dashed lines.

In Fig. $1 \mathrm{~b}$ one can find the phase $\varphi$ development in time. The phase $\varphi$ can be approximated by straight lines with negative slopes during the investigated group intervals. It is obvious that the local frequency $\omega(t)$ of the largest wave groups is smaller than the calculated carrier frequency $\omega_{0}$ of the series because $\mathrm{d} \varphi(t) / \mathrm{d} t<0$. Between the high groups there are time intervals when the amplitudes are very small and a significant positive phase change is registered which is manifested by jumps of $\varphi$. Such jumps have been observed previously in Guedes Soares and Cherneva (2005) for the waves in deep water near the Portuguese coast. Studying the evolution of nonlinear wave trains, Melvill (1983) first suggests that "crest pearing" (Ramamonjiarisoa and MolloCristensen, 1979; Mollo-Cristensen and Ramamonjiarisoa, 1982) may appear as a result of large positive gradients in phase speed when one crest overtakes the previous.

In the left-side panels in Figs. 2-4 the spectrum of the whole series with peak frequency $\omega_{\mathrm{p}}=0.475 \mathrm{rad} \mathrm{s}^{-1}$ calculated by the well-known Welch method (Welch, 1967) is presented. The time-frequency spectra of the three groups from Fig. 1 are drawn in 10 different colours on the right side of the figures. To present the Wigner spectrum, the surface of the energy distribution is normalized to its maximum value. Ten colours are used to show the Wigner spectrum, meaning that the "resolution" by colour is $10 \%$ of the maximum value. For the sake of convenience the waves are indicated in white at the same timescale as the time-frequency 

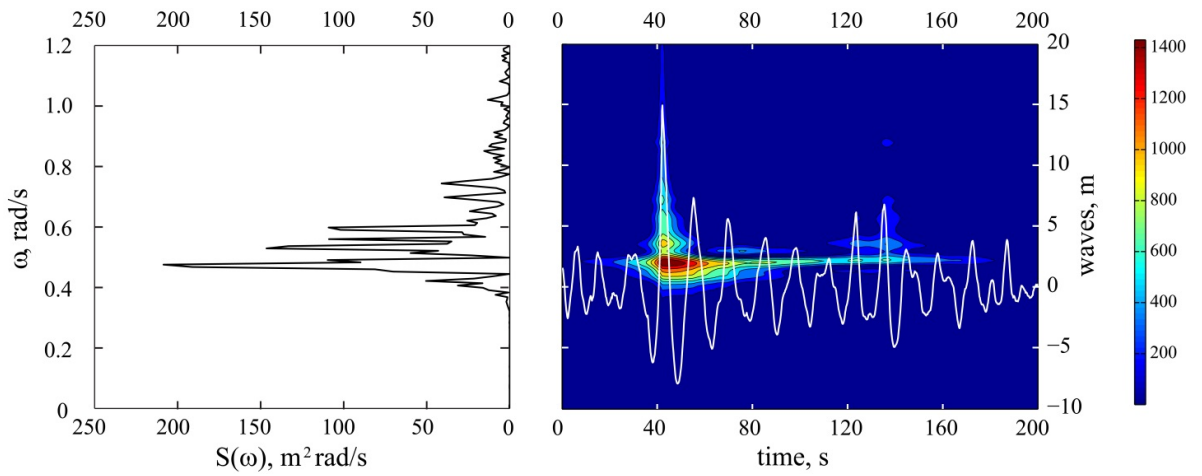

Figure 2. Time-frequency spectrum of group I containing the freak wave.
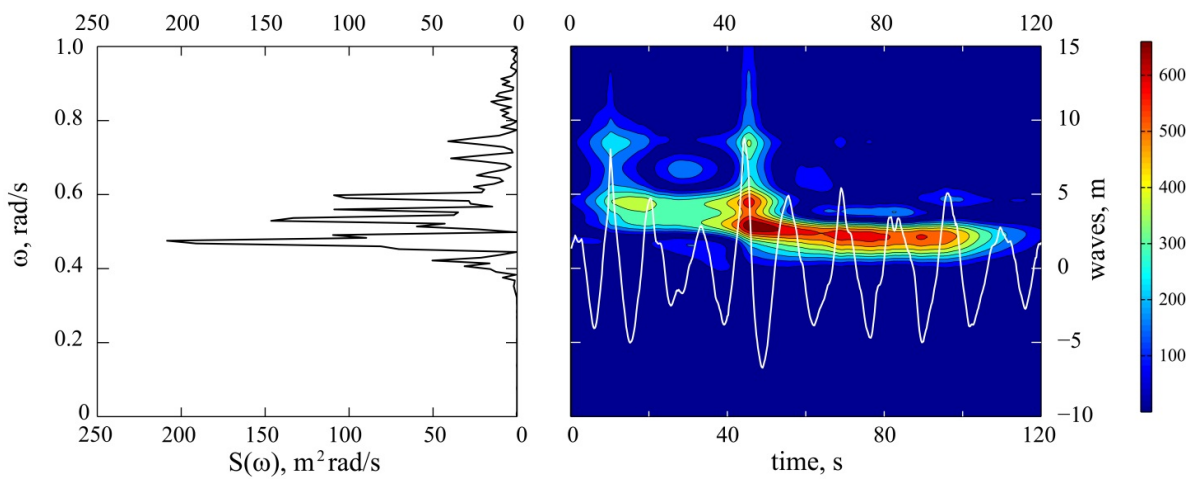

Figure 3. Time-frequency spectrum of group II between 440 and $520 \mathrm{~s}$.

distribution. Spectrum and time-frequency spectra are calculated with same frequency resolution.

The Wigner spectrum of the group containing the Andrea freak wave is presented in Fig. 2. During the abnormal wave the energy is distributed in a wide frequency interval. There are several peaks that coincide with the corresponding peaks of the usual frequency spectrum. The group has the typical triangle form for the nonlinear waves.

Investigating the nonlinear high waves and the group development in the tank, the triangle group form has been registered many times (Cherneva and Guedes Soares, 2012). The form is a result of continuous shrinking of the group at different distances from the wave maker and of changing the place of the highest wave from the middle to the front of the group. For the tank-produced waves, the process is accompanied with frequency downshifting of the energy peak inside the group demonstrated by the time-frequency spectrum. The group is extremely short at the point of focus and has a form similar to the New Year wave group. Moving forward, the focus the group disintegrates and the time-frequency spectrum shows frequency upshifting of the energy peak. The same character of group development with distance is noted by Kharif et al. (2008) in their investigation of the wind's influence on the wave groups at different wind velocities $U$ (including $U=0$ ) and distances.
It has to be remarked that there is no registered downshifting of the energy maximum of the time-frequency spectrum of the group containing the Andrea abnormal wave. In a spectrogram analysis of real sea states it was also noticed that not all groups demonstrate frequency downshifting of the energy. A similar conclusion was obtained by Su (1982), who investigated the gravity wave group evolution with moderate and high steepness.

It is also necessary to mention that a frequency upshifting like the one for the groups in the tank after the focus is not registered in real sea states.

Frequency downshifting of the energy peak is demonstrated in Fig. 3, which presents the time-frequency spectrum of the series between 440 and $520 \mathrm{~s}$ (see Fig. 1a). Again, during the highest wave of the group the energy spreads in a wide frequency interval with three dominating peaks. The peak that prevails over two other peaks has a frequency higher than the $\omega_{p}$ of the usual spectrum. The energy peak that towers in further waves in the group slowly moves to lower frequencies and reaches the place of $\omega_{\mathrm{p}}$. If the same scenario, as explained before for the in-tank generated nonlinear waves, takes place for the examined wave group, it is possible that it will transform into a short group similar to that of the New Year wave after several wavelengths. 

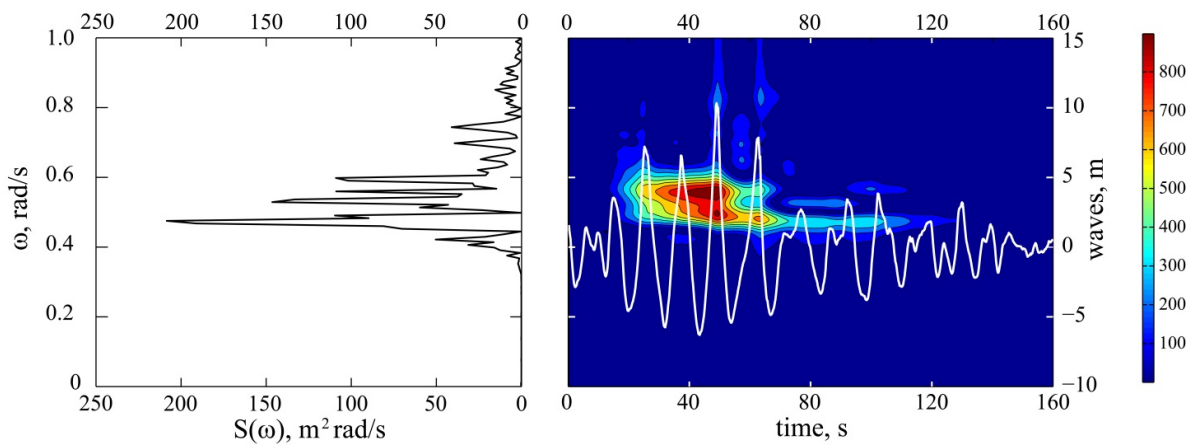

Figure 4. Time-frequency spectrum of group III between 130 and $235 \mathrm{~s}$.

Figure 4 shows the Wigner spectrum of the group that exists in the time interval of 130-235 s (see Fig. 1a). The phase rate for that group is not constant and slowly changes in time. The picture of the peaks registered in the time-frequency spectrum of the group is very interesting. The first three waves have heights of less than $3 \mathrm{~m}$. Their energy peaks are less than $10 \%$ of the highest peak during the group's lifetime and are invisible. The fourth and the fifth waves have the energy peak of the same frequency. The energy of that peak increases with time. It reaches its maximum value during the highest wave of the group and drastically decreases for the next waves. A second peak of lower frequency than the first one during the highest wave appears. It begins to dominate the energy distribution in the lifetime of the next waves. Weak downshifting of that second peak is observed since it reaches the frequency $\omega_{\mathrm{p}}$. A similar type of frequency downshifting during the group's lifetime has already been observed (Guedes Soares and Cherneva, 2005) for a two-wave system sea state without abnormal waves, a broad spectrum and a small angle between the main directions of the system. The peak in the higher frequency of one wave system shifts to the low frequency and unites with the peak of the second wave system. It can be suggested that the complicated time-frequency spectrum picture of group III in Fig. $1 \mathrm{~b}$ is possibly a result of the interaction of wave components coming from separate directions. As Magnusson and Donelan (2013) note, "the Andrea wave is observed just past 00 UTC, after a stable period with less wind forcing, but at the start of a new wind increase from a new and slightly different direction".

\section{Conclusions}

The example of the Andrea series was measured in harsh meteorological conditions and has a higher steepness than previously analysed waves by Guedes Soares and Cherneva (2005). The abnormal Andrea wave exists in a large group of 12 waves and differs from the New Year wave which exists in a group similar to the well-known "three sisters".
Here, the time-frequency analysis is made with a more sophisticated Wigner spectrum but shows a similar character of energy distribution during the group's lifetime as that traced out by spectrogram analyses. It is revealed that during the highest wave of the group, the energy spreads in a wide frequency interval with several peaks and confirms the previous results for the abnormal Draupner wave (Cherneva and Guedes Soares, 2008).

The abnormally high Andrea wave is registered in a wave group having a constant local frequency. That local frequency $\omega(t)$ is less than the calculated carrier frequency $\omega_{0}$ of all series using Eq. (4). In time, the abnormal wave happens at exactly the moment in which the series' highest peak of energy exists and has frequency equal to the frequency $\omega_{\mathrm{p}}$ of the usual stationary spectrum.

Not all groups investigated in real sea states demonstrate a frequency downshifting of the energy with time. For example, the long group that contains the Andrea freak wave has no frequency downshifting of that type registered in the Marintek tank. Also, frequency upshifting like that marked after the focus for the in-tank groups is not registered in real sea states investigated by the authors.

The energy distribution in time of the groups that have a changeable local frequency $\omega(t)$ is more sophisticated. The waves in such groups are not very steep, but in the time interval when the highest waves exist a significant downshifting of the local spectrum peak is observed. It is realized by a jump during the highest wave. It is suggested that more complicated downshifting is possibly a consequence of the interaction of wave components coming from separate directions.

Acknowledgements. The present work had been partly supported by EU contract no. SCP8-GA-2009-234175, EXTREME SEAS Design for Ship Safety in Extreme Seas.

Edited by: E. Bitner-Gregersen

Reviewed by: five anonymous referees 


\section{References}

Benjamin, T.: Instability of periodic wave trains in nonlinear dispersive systems, P. Roy. Soc. Lond. A, 299, 59-76, 1967.

Benjamin, T. and Feir, J.: The Disintegration of Wave Trains on Deep Water, Part I, J. Fluid Mech., 27, 417-430, 1967.

Bitner, E.: Non-linear effects of the statistical model of shallow water wind waves, Appl. Ocean Res., 2, 63-73, 1980.

Bitner-Gregersen, E. and Gran, S.: Local properties of sea waves defined from a wave record, Appl. Ocean Res., 5, 210-214, 1983.

Boashash, B.: Time-Frequency Signal Analysis, in: Advances in Spectrum Estimation, edited by: Haykin, S., Englewood Cliffs, Prentice Hall, NJ, 418-517, 1991.

Boashash, B. and Whitehouse, H.: Seismic applications of the Wigner-Wille distribution, Proc. IEEE Conf. Systems and Circuits, San Jose, USA, 34-37, 1986.

Boles, P. and Boashash, B.: The cross Wigner-Ville distribution - a two-dimensional analysis method for the processing of vibroseis seismic signals, Proc. IEEE ICASSP, 87, 904-907, 1988.

Cherneva, Z. and Guedes Soares, C.: Bispectra and time-frequency spectra of wind waves in the coastal zone, in: Maritime Transportation and Exploitation of Ocean and Coastal Resources, edited by: Guedes Soares, C., Garbatov, J., and Fonseca, N., Taylor and Francis Group, London, 1005-1014, 2005.

Cherneva, Z. and Guedes Soares, C.: Non-linearity and nonstationarity of the New Year abnormal wave, Appl. Ocean Res., 30, 215-220, 2008.

Cherneva, Z. and Guedes Soares, C.: Non-linear and Non-stationary sea waves, in: Maritime Transportation and Exploitation of Ocean and Coastal Resources, edited by: Guedes Soares, C., Garbatov, Y., Fonseca, N., and Teixeira, A. P., Taylor and Francis Group, London, 45-67, 2011.

Cherneva, Z. and Guedes Soares, C.: Non-Gaussian wave groups generated in an offshore basin, J. OMAE, 134, 041602-1, doi:10.1115/1.4006394, 2012.

Cherneva, Z., Tayfun, M. A., and Guedes Soares, C.: Statistics of nonlinear waves generated in a offshore basin. J. Geoph. Res., 14, C08005, doi:10.1029/2009JC005332, 2009.

Cherneva, Z., Tayfun, M. A., and Guedes Soares, C.: Statistics of waves with different steepness symulated in a wave basin, Ocean Eng., 60, 186-192, 2013.

Choi, H. and Williams, W.: Improved Time-frequency Representation of Multicomponent Signals Using Exponential Kernels, IEEE T. Acoust. Speech Sign Proc., 37, 862-871, 1999.

Claasen, T. and Meeklenbräuker, W.: The Wigner distribution - a tool for time-frequency signal analysis, Part I: continuous-time signals, Phillips J. Res., 35, 217-250, 1980a.

Claasen, T. and Meeklenbräuker, W.: The Wigner distribution - a tool for time-frequency signal analysis, Part II: discrete time signals, Phillips J. Res., 35, 276-300, 1980b.

Claasen, T., and Meeklenbräuker, W.: The Wigner distribution - a tool for time-frequency signal analysis, Part III; relations with other time-frequency signal transformations, Phillips J. Res., 35, 372-389, 1980c.

Cohen, L.: Generalised phase-space distribution functions, J. Math. Phys., 7, 781-786, 1966.

Cohen, L.: Time frequency distributions. A review, Proc. IEEE, 77, 941-981, 1989.

Cohen, L.: Time-frequency Signal Analysis, Prentice-Hall, Englewood Cliffs, NJ, p. 299, 1995.
Didenkulova, I.: Shapes of freak waves in the coastal zone of the Baltic Sea (Tallin Bay), Boreal Environ. Res., 16, 138-148, 2011.

Faulkner, D. and Buckley, W. H.: Critical Survival Conditions for Ship Design, paper no. 6, International Conference on Design and Operation for Abnormal Conditions, RINA, London, UK, 1-25, 1997.

Fonolosa, J. and Nikias, C.: Wigner Higher Order Moment Spectra: Definition, Properties, Computation and Application to Transient Signal Analysis, IEEE T. Sign. Proc., 41, 245-266, 1993.

Fonolosa, J. and Nikias, C.: Analysis on finite-energy signals using higher-order moments and spectra-based time-frequency distributions, Signal Proc., 36, 315-328, 1994.

Gabor, D.: Theory of communication, J. IEE Lond., 93, 429-457, 1946.

Guedes Soares, C. and Cherneva, Z.: Spectrogram analysis of the time-frequency characteristics of ocean wind waves, Ocean Eng., 32, 1643-1663, 2005.

Guedes Soares, C. Cherneva, Z., and Antão, E.: Characteristics of abnormal waves in North Sea storm sea states, Appl. Ocean Res., 25, 337-344, 2003.

Guedes Soares, C., Cherneva, Z., and Antão, E.: Abnormal waves during the hurricane Camille, J. Geophys. Res., 109, C08008, doi:10.1029/2003JC002244, 2004.

Hammack, J. L. and Henderson, D.: Resonant interactions among surface water waves, Ann. Rev. Fluid Mech., 25, 55-97, 1993.

Haver, S. and Andersen, O. J.: Freak Waves: Rare Realizations of a Typical Population or Typical Realizations of a Rare Population?, Proc. 10th Int. Offshore and Polar Eng. Conf., 28 May-2 June, Seattle, USA, 123-130, 2000.

Hlawatsch, F. and Boudreaux-Bartels, G.: Linear and Quadratic Time-Frequency Signal Representations, IEEE Signal Proc. Mag., 9, 21-67, 1992.

Hlawatsch, F. and Flandrin, P.: The Interference structure of the Wigner distribution and related time-frequency signal representations, in: Wigner distribution - Theory and applications in signal processing, edited by: Mecklenbrauker, W., North Holland Elsevier Science Publishers, Amsterdam, the Netherlands, 1992.

Huang, N., Shen, Z., Long, S., Wu, M., Shih, H., Zheng, Q., Yen, N., Tung, C., and Liu, H.: The empirical mode decomposition and the Hilbert spectrum for non-linear and non-stationary time series analysis, P. Roy. Soc. Lond. A, 454, 903-995, 1998.

Janse, C. and Kaizer, M.: Time-frequency distributions of loudspeakers: the application of the Wigner distribution, J. Audio Eng. Soc., 31, 198-223, 1983.

Kharif, C. Giovanangeli, J.-P., Touboul, J., Grare, L., and Pelinovsky, E.: Influence of wind on extreme wave events: experimental and numerical approaches, J. Fluid Mech., 594, 209-247, 2008.

Kharif, C. Pelinovsky, E., and Slunyaev, A.: A Rogue Waves in the Ocean, Springer-Verlag, Berlin, Heidelberg, p. 216, 2009.

Kjeldsen, S.: Examples of Heavy Weather Damages caused by Giant Waves, B. Soc. Naval Arch. Jpn., 828, 744-748, 1997.

Krasitskii, V.: On reduced equations in the Hamiltonian theory of weakly nonlinear surface waves, J. Fluid Mech., 272, 1-30, 1994.

Kurkin, A. and Pelinovsky, E.: Freak waves: Facts, theory and modeling, Nizhegorod Techn. Univ. Printing House, N. Novgorod, p. 158, 2004. 
Lake, B., Yuen, H., Rungaldier, H., and Ferguson, W.: Non-linear deep water waves: Theory and experiment, Part 2, Evolution of a continuous wave train, J. Fluid Mech., 83, 49-74, 1977.

Levin, M.: Instantaneous spectra and ambiguity functions, IEEE T. Informat. Theory, 13, 95-97, 1967.

Liu, P.: Wavelet spectrum analysis and ocean wind waves, in: Wavelets in Geophysics, edited by: Foufoula-Georgiou, E. and Kumar, P., Acad. Press, New York, 151-166, 1994.

Liu, P.: Is the wind wave frequency spectrum outdated, Ocean Eng., 27, 577-588, 2000a.

Liu, P.: Wave grouping characteristics in nearshore Great Lakes, Ocean Eng., 27, 1221-1230, 2000b.

Longuet-Higgins, M. S.: On the statistical distribution of the heights of sea waves, J. Mar. Res., XI, 245-266, 1952.

Longuet-Higgins, M. S.: The instabilities of gravity waves of finite amplitude in deep water, II. Subharmonics, P. Roy. Soc. Lond. A, 360, 489-505, 1978.

Magnusson, A. and Donelan, M.: The Andrea Wave Characteristics of a Measured North Sea Rogue Wave, J. OMAE, 135, 031108-1, doi:10.1115/1.4023800, 2013.

Mark, W.: Spectral analysis of the convolution and filtering of nonstationary stochastic processes, J. Sound Vib., 11, 19-63, 1970.

Massel, S.: Wavelet analysis for processing of ocean surface wave records, Ocean Eng., 28, 957-987, 2001.

Massel, S.: Surface waves in deep and shallow waters, Oceanologia, Gdansk, 52, 5-52, 2010.

Meecklenbräuker, W.: A Tutorial on Non-Parametric Bilinear Time Frequency Signal Representations, in: Les Houches, session XLV, Signal Processing, edited by: Lacoume, J., Durani, T., and Stora, R., Amsterdam, Oxford, New York, North-Holland, 277-336, 1985 .

Melvill, W.: Wave modulation and breakdown, J. Fluid Mech., 128, 989-506, 1983.

Mollo-Christensen, E. and Ramamonjiarisoa, A.: Subharmonic transitions and group formation in a wind wave field, J. Geophys. Res., 87, 5699-5717, 1982.

Mori, N., Liu, P., and Yasuda, T.: Analysis of Freak Wave Measurements in the Sea of Japan, Ocean Eng., 29, 1399-1414, 2002.

Page, C.: Instantaneous power spectra, J. Appl. Phys., 23, 103-106, 1952.

Ramamonjiarisoa, A. and Mollo-Christensen, E.: Modulation characteristics of sea surface waves, J. Geophys. Res., 84, 7769-7775, 1979.

Rihaczek, W.: Signal energy distribution in time and frequency, IEEE T. Informat. Theory., 14, 369-374, 1968.

Schurlmann, T.: Spectral frequency analysis of nonlinear water waves based on the Hilbert-Huang transformation, Proc. OMAE'01, 20th Int. Conf on Offshore Mech. And Arctic Eng., 3-8 June 2001, Rio de Janeiro, Brasil, 2001.
Skourup, J. K., Andreassen, K., and Hansen, N. E. O.: NonGaussian Extreme Waves in the Central North Sea, Proc. OMAE'1996, Part A, ASME, New York, 1996.

$\mathrm{Su}, \mathrm{M} .-\mathrm{Y}$.: Evolution of groups of gravity waves with moderate to high steepness, Phys. Fluids, 25, 2167-2174, 1982.

Tayfun, M. A. and Lo, J.: Envelope, Phase and Narrow-band Models of Sea Waves, J. Waterway Port Coast Ocean Eng.-ASCE, 115, 594-613, 1989.

Tulin, M. P. and Waseda, T.: Laboratory observations of wave group evolution including breaking effects, J. Fluid Mech., 378, 197-232, 1999.

Turner, C.: On the concept of an instantaneous spectrum and its relationship to the autcorrelation function, J. Appl. Phys., 25, 1347-1351, 1954.

Van der Pol, B.: The fundamental principles of frequency modulation, J. IEE Lond., 93, 153-158, 1946.

Veltcheva, A. and Guedes Soares, C.: Analysis of Abnormal Wave Records by the Hilbert Huang Transform Method, J. Atmos. Ocean. Tech., 24, 1678-1689, 2007.

Veltcheva, A. and Guedes Soares, C.: Application of the HilbertHuang Transform analysis to sea waves, in: Maritime Transportation and Exploitation of Ocean and Coastal Resources, edited by: Guedes Soares, C., Garbatov, Y., Fonseca, N., and Teixeira, A. P., Taylor and Francis Group, London, 45-67, 2011.

Ville, J.: Theorie et applications de la notion de signal analitique, Cables et Transmission, 2a, 61-74, 1948.

Welch, P.: The use of Fast Fourier Transform for the estimation of power spectra: a method based on time averaging over short, modified periodograms, IEEE T. Audio Electroacoust., AU-15, 70-73, 1967.

Wigner, E.: On the quantum correction for thermodynamic equilibrium, Phis. Rev., 40, 749-759, 1932.

Wolfram, J., Linfoot, B., and Stansell, P.: Long- and short-term extreme wave statistics in the North Sea (1994-1998), Rogue Waves, edited by: Olagnon, M. and Athanassoulis, G. A., 2000, 363-372, 2000.

Yasuda, T. and Mori, N.: Occurrence properties of giant freak waves in the sea area around Japan, J. Waterway Port Coast. Ocean Eng., 123, 209-213, 1997.

Yuen, H. and Lake, M.: Instabilities of waves on deep water, Ann. Rev. Fluid Mech., 12, 303-334, 1980.

Zakharov, V.: Stability of periodic waves of finite amplitude on the surface of a deep fluid, J. Appl. Mech. Tech. Phys., 9, 190-194, 1968. 